\title{
In humans, early cortisol biosynthesis provides a mechanism to safeguard female sexual development
}

\author{
Masahiro Goto, ${ }^{1}$ Karen Piper Hanley, ${ }^{1,2}$ Josep Marcos, ${ }^{3}$ Peter J. Wood, ${ }^{4}$ Sarah Wright, ${ }^{1}$ \\ Anthony D. Postle, ${ }^{5}$ lain T. Cameron, ${ }^{2,6}$ J. Ian Mason, ${ }^{7}$ David I. Wilson,, ${ }^{1,2}$ and Neil A. Hanley ${ }^{1,2}$ \\ ${ }^{1}$ Human Genetics Division and ${ }^{2}$ Early Human Development and Stem Cells Group, University of Southampton, Southampton, United Kingdom. \\ 3Pharmacology Research Unit, Institut Municipal d'Investigació Médica, Department of Experimental and Health Sciences, Pompeu Fabra University, Barcelona, \\ Spain. ${ }^{4}$ Department of Chemical Pathology, Southampton University Hospitals National Health Service Trust, Southampton, United Kingdom. \\ 5Inflammation, Infection, and Repair Division and 'Developmental Origins of Health and Disease Division, University of Southampton, Southampton, \\ United Kingdom. ${ }^{7 R e p r o d u c t i v e ~ a n d ~ D e v e l o p m e n t a l ~ S c i e n c e s, ~ C e n t r e ~ f o r ~ R e p r o d u c t i v e ~ B i o l o g y, ~ U n i v e r s i t y ~ o f ~ E d i n b u r g h, ~ E d i n b u r g h, ~ U n i t e d ~ K i n g d o m . ~}$
}

In humans, sexual differentiation of the external genitalia is established at 7-12 weeks post conception (wpc). During this period, maintaining the appropriate intrauterine hormone environment is critical. In contrast to other species, this regulation extends to the human fetal adrenal cortex, as evidenced by the virilization that is associated with various forms of congenital adrenal hyperplasia. The mechanism underlying these clinical findings has remained elusive. Here we show that the human fetal adrenal cortex synthesized cortisol much earlier than previously documented, an effect associated with transient expression of the orphan nuclear receptor nerve growth factor IB-like (NGFI-B) and its regulatory target, the steroidogenic enzyme type 2 $3 \beta$-hydroxysteroid dehydrogenase (HSD3B2). This cortisol biosynthesis was maximal at 8-9 wpc under the regulation of ACTH. Negative feedback was apparent at the anterior pituitary corticotrophs. ACTH also stimulated the adrenal gland to secrete androstenedione and testosterone. In concert, these data promote a distinctive mechanism for normal human development whereby cortisol production, determined by transient NGFI-B and HSD3B2 expression, provides feedback at the anterior pituitary to modulate androgen biosynthesis and safeguard normal female sexual differentiation.

\section{Introduction}

The differentiation of male or female phenotype consistent with chromosomal sex is a key component of development. In humans, sexual dimorphism of the external genitalia is established during a critical phase of the first trimester in response to male hormones from the fetal testis. During this period, overexposure of the 46,XX female fetus to these androgens causes virilized genitalia at birth. Most commonly, this results from congenital adrenal hyperplasia due to deficiency of cytochrome P450 21-hydroxylase (CYP21; Figure 1), an enzyme in the biosynthetic pathway to cortisol (1). Lack of CYP21 and, consequently, cortisol is predicted to relieve negative feedback at the fetal anterior pituitary so that excess ACTH drives steroid precursors toward androgen biosynthesis (Figure 1). This balance between cortisol biosynthesis and potential androgen production is emphasized by the restoration of normal female development in CYP21 deficiency if the synthetic glucocorticoid dexamethasone, which crosses the placenta, is administered to the mother by 6-7 weeks post conception (wpc) $(1,2)$.

These clinical data generate a clear hypothesis. However, direct evidence of adrenocortical activity during the first trimester, and its potential to impact on sexual differentiation, is hampered by restricted access to human fetal material and compounded by the

Nonstandard abbreviations used: CYP21, cytochrome P450 21-hydroxylase; $\mathrm{dpc}$, days post conception; GR, glucocorticoid receptor; HSD17B,

$17 \beta$-hydroxysteroid dehydrogenase; HSD3B, $3 \beta$-hydroxysteroid dehydrogenase; IHC, immunohistochemistry; NGFI-B, nerve growth factor IB-like; StAR, steroid acute regulatory protein; wpc, weeks post conception.

Conflict of interest: The authors have declared that no conflict of interest exists. Citation for this article: J. Clin. Invest. 116:953-960 (2006). doi:10.1172/JCI25091. unique nature of the developing adrenal cortex in higher primates $(3,4)$. The available data are inconclusive. In particular, first-trimester cortisol is assumed to maintain negative feedback upon ACTH secretion. However, an important enzyme for cortisol biosynthesis, type $23 \beta$-hydroxysteroid dehydrogenase (HSD3B2), has only been detected late in the second trimester $(3,5)$ (Figure 1 ). Although ACTH at least partially regulates adrenal steroidogenesis after $12 \mathrm{wpc}(3)$, earlier immunoreactivity in the human fetal anterior pituitary (6) has never been correlated to adrenal function. Indeed, anencephalic fetuses, which lack the anterior pituitary, have normal appearing adrenals during the first trimester (7).

For these reasons, we sought to clarify the potential for the adrenal cortex to participate in cortisol and androgen biosynthesis during the first trimester of human development. In this study, we determined that adrenocortical activity correlated to that of the developing anterior pituitary. Our findings, linked to transient expression of the key steroidogenic enzyme HSD3B2 and its regulatory orphan nuclear receptor, nerve growth factor IB-like (NGFI-B) (8), support a distinctive developmental mechanism for cortisol biosynthesis in humans that protects normal female sexual differentiation.

\section{Results}

The human adrenal cortex is distinct from the bipotential gonad at 33 days post conception $(\mathrm{dpc})(9,10)$, after which it can be dissected free from surrounding structures, including the kidney (Figure 2A). The adrenal cortex grew rapidly during the early fetal period. Its wet weight increased almost 10 -fold from $8 \mathrm{wpc}$ to $10 \mathrm{wpc}$ (Figure 2B). At $41 \mathrm{dpc}$ CD34-positive vascular channels 


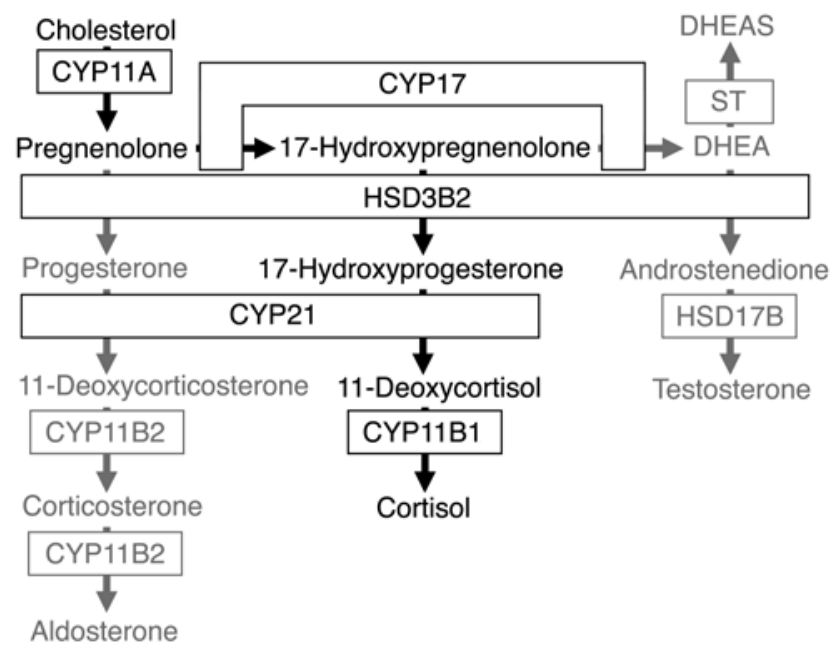

Figure 1

Steroidogenic pathways involving the human adrenal cortex. De novo cortisol biosynthesis is shown in black; other pathways are in gray. DHEA, dehydroepiandrosterone; DHEAS, DHEA sulfate; ST, sulfotransferase.

penetrated the embryonic gland between the aorta and mesonephros, and vessel density later increased at the adrenocortical periphery (Figure 2, C and D).

The precise onset of adrenocortical function has not been defined, either by steroid secretion or expression profile of the biosynthetic enzymes (Figure 1). By immunohistochemistry (IHC) as shown in Figure 3, steroid acute regulatory protein (StAR), CYP11A, CYP17, and CYP21 were first detected robustly at 50-52 dpc within the nascent inner fetal zone (Figure 3, B, G, L, and Q). Immunoreactivity in this location was also detected using an antibody that recognizes both CYP11B1 and CYP11B2 (Figure 3V). Using specific primers and sequencing, RT-PCR amplified products from both CYP11B1 and CYP11B2 transcripts (data not shown). Within the outer definitive zone, StAR, CYP11A, CYP21, and CYP11B1/CYP11B2 were more weakly detected and, in line with our previous investigations, CYP17 appeared largely absent
(Figure 3L) (10). In later specimens up to $14 \mathrm{wpc}$, these profiles persisted in the fetal zone; however, the definitive zone also stained weakly positive for CYP17 (Figure 3, M, N, and O).

For de novo cortisol biosynthesis from cholesterol, the expression of HSD3B2 is necessary (Figure 1). In all previous studies restricted to later material, this protein has only been detected reliably after 22 weeks' gestation $(3,5)$, where it is regulated by the orphan nuclear receptor NGFI-B (8). Like other adrenocortical enzymes, HSD3B immunoreactivity was absent at $41 \mathrm{dpc}$; yet surprisingly, positive cells were apparent at 50-52 dpc, mostly at the interface between the definitive and fetal zones (Figure 4, A and B). Expression was particularly abundant and more widespread in specimens at 8-9 wpc, with many cells demonstrating HSD3B immunoreactivity that declined at later time points until no protein could be detected at $14 \mathrm{wpc}$ (Figure 4, C-F). IHC for NGFI-B on neighboring sections revealed a remarkably similar profile of nuclear expression (primary antibodies to HSD3B and NGFI-B were both raised in rabbit prohibiting colocalization studies; Figure 4, G-L).

For all the IHC experiments, expression was indistinguishable between male and female specimens. The data were also supported by sequenced RT-PCR products for StAR, CYP11A, CYP17, CYP21, and $H S D 3 B$ isoforms using intron-spanning primers (Figure $5 \mathrm{~A}$ ). The HSD3B immunoreactivity correlated solely to expression of the type 2 isoform HSD3B2 (Figure 5B).

Our findings on enzyme expression predicted cortisol biosynthesis. Compared with the kidney, immunoassays for cortisol, validated by gas chromatography/mass spectrometry (Supplemental Methods and Supplemental Figure 1; supplemental material available online with this article; doi:10.1172/JCI25091DS1), revealed 9- to 18-fold higher cortisol content in the adrenal gland during the first trimester (Figure 6A). Although this steep gradient could reflect differential metabolism, adrenal expression of type 2 $11 \beta$-hydroxysteroid dehydrogenase (HSD11B2), which inactivates cortisol to cortisone, exceeded that in the early kidney (data not shown). Cortisol content per adrenal weight dropped by approximately $50 \%$ between 8 and $10 \mathrm{wpc}$ (Figure $6 \mathrm{~A}$ ), consistent with the waning profile of HSD3B2 expression (Figure 4).

The response of paired first-trimester adrenal glands to agonist was investigated in male and female specimens by overnight cul-

\section{Figure 2}

Growth and vascular development of the human early fetal adrenal gland. (A) Relative proportion of adrenal gland (ADR) to kidney (KID) during early development. (B) Weight ( \pm SEM) of individual adrenal glands and developmental age. (C and D) Brightfield IHC with anti-CD34 to demonstrate vascular development counterstained by toluidine blue at 41 (C) and 50 (D) dpc. An adjacent H\&E section is shown at the left of $\mathbf{C}$. ac, adrenal cortex; ao, aorta; c, capsule; mg, mesonephric glomerulus. Scale bars: $1 \mathrm{~mm}(\mathbf{A})$, $300 \mu \mathrm{m}$ (C and D).
A
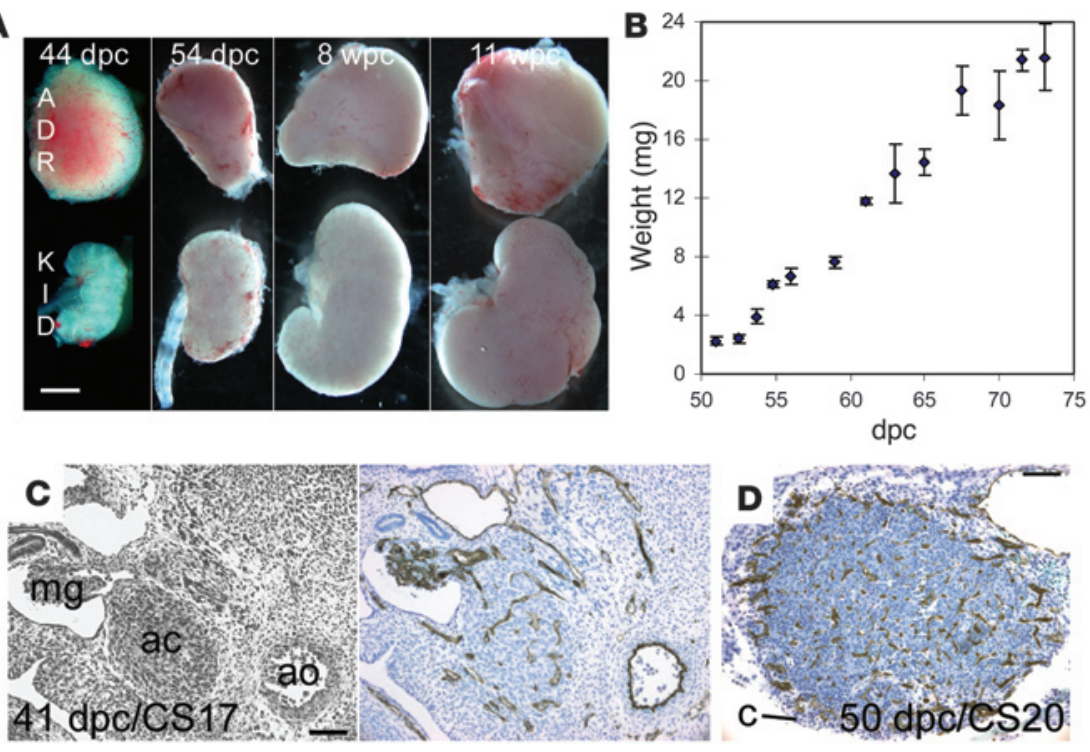

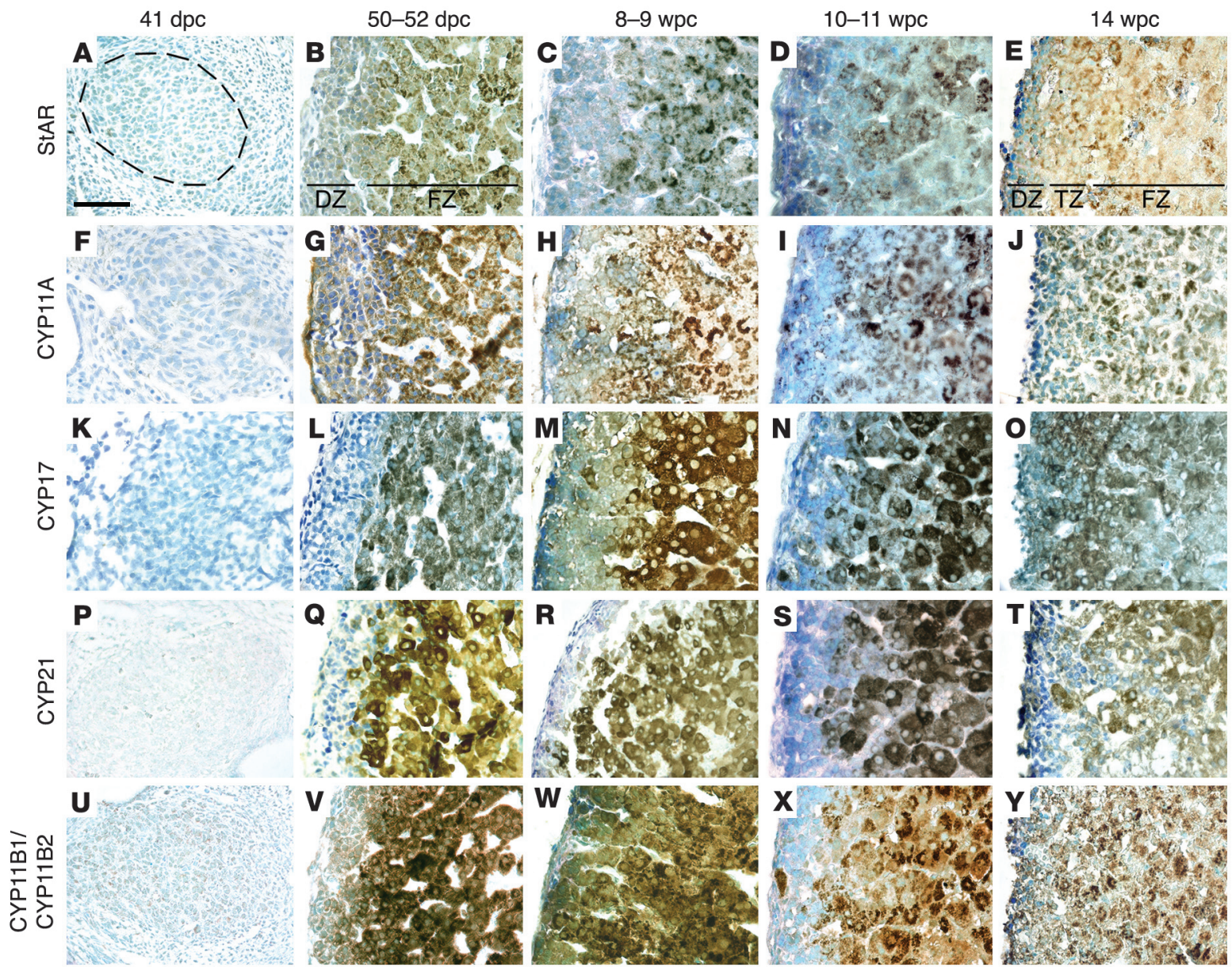

Figure 3

Steroidogenic enzyme and StAR expression in the developing human adrenal gland. Brightfield IHC at sequential developmental ages counterstained by toluidine blue with antibodies to StAR (A-E), CYP11A (F-J), CYP17 (K-0), CYP21 (P-T), and CYP11B1/CYP11B2 (U-Y). Dotted ring in $\mathbf{A}$ illustrates extent of the adrenal cortex in left panels ( $\mathbf{A}, \mathbf{F}, \mathbf{K}, \mathbf{P}$, and $\mathbf{U})$. In other images, the definitive zone (DZ) is oriented to the left and the fetal zone (FZ) to the right. At $14 \mathrm{wpc}$, these 2 zones were separated by an additional transitional zone (TZ). Scale bar: $300 \mu \mathrm{m}$.

ture and media assay for cortisol. Ex vivo culture of human adrenocortical cells at mid-gestation has artificially induced HSD3B2 (3). RT-PCR before and after culture ensured that this was not the case in our experiments (Supplemental Figure 2). Forskolin, which mimics the effect of ACTH, stimulated an 11-fold increase in adrenal cortisol content at 8 and 9 wpc compared with only 7.5 -fold at
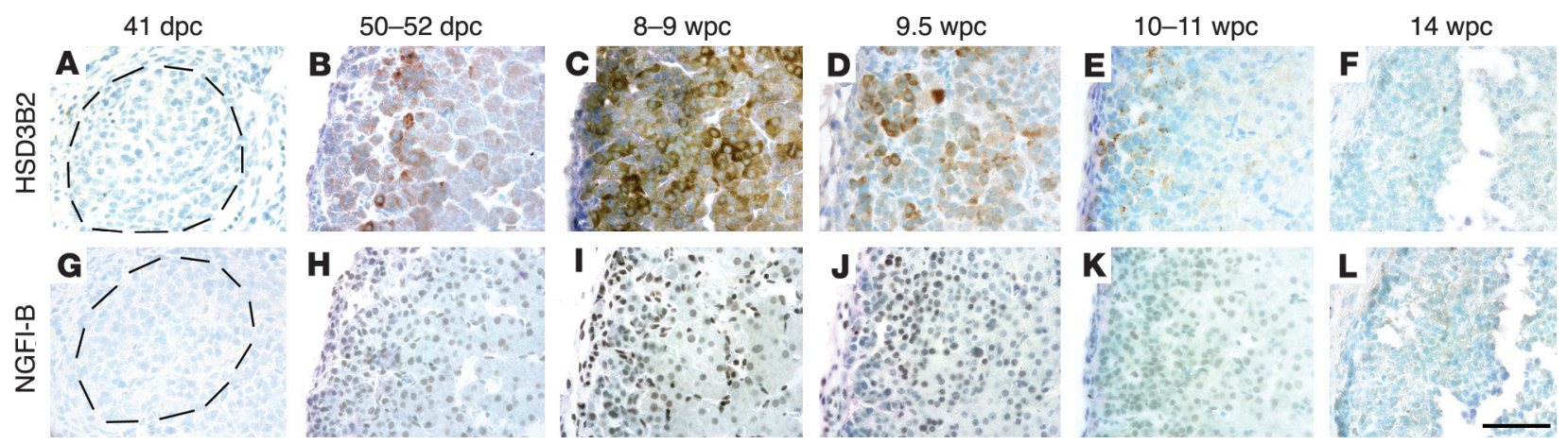

Figure 4

HSD3B and NGFI-B immunoreactivity in the developing human adrenal gland. Brightfield IHC with antibodies to HSD3B (A-F) and NGFI-B (G-L) counterstained with toluidine blue. Dotted rings in $\mathbf{A}$ and $\mathbf{G}$ illustrate extent of the adrenal cortex. In other images, the definitive zone is oriented to the left and fetal zone to the right. Scale bar: $300 \mu \mathrm{m}$. 

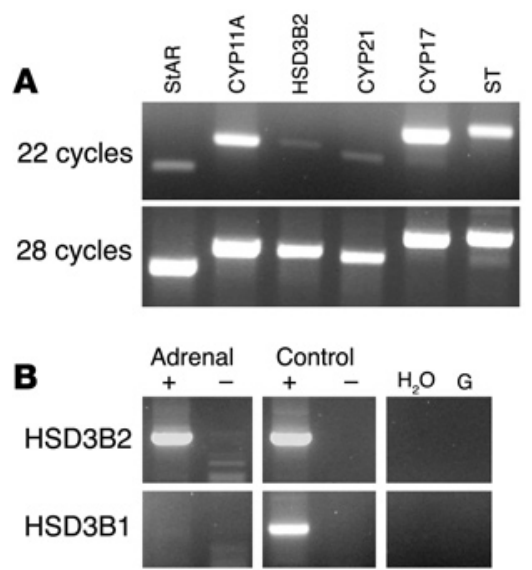

$10 \mathrm{wpc}$ (data not shown). Very similar results were obtained from the media, showing significantly diminished cortisol secretion at $10 \mathrm{wpc}$ (Figure 6B). These data defined maximal cortisol production at $8-9 \mathrm{wpc}$, when the adrenal gland was also responsive to ACTH (Figure 6C) and expressed its receptor, the type 2 melanocortin receptor (MC2R; Figure 6D).

Collectively, these experiments confirmed ACTH-responsive cortisol biosynthesis by the early human adrenal cortex. We used the same fetal collection to ask whether there was coincident maturity

\section{Figure 5}

RT-PCR analysis of adrenocortical enzymes and StAR at 8 wpc. (A) RT followed by 22 and 28 cycles of PCR for transcripts encoding steroidogenic enzymes. (B) Specific identification of HSD3B2 and HSD3B1 isoforms in the presence $(+)$ and absence $(-)$ of RT. No HSD3B1 transcript was detected in the adrenal sample after 42 cycles of PCR. Positive controls were fetal testis (HSD3B2) and skin (HSD3B1); negative controls were $\mathrm{H}_{2} \mathrm{O}$ and genomic DNA (G).

of anterior pituitary corticotrophs to secrete ACTH. The developing pituitary was negative for ACTH immunoreactivity at $41 \mathrm{dpc}$ (data not shown). However, identical to the onset of adrenocortical enzyme expression, cytoplasmic ACTH was detected at 50-52 dpc (Figure 7B), which overlapped with the more widespread expression of nuclear glucocorticoid receptor (GR; Figure 7C; note that colocalization studies were not possible as both antibodies were raised in mice). Increased ACTH expression was detected in the anterior pituitary at $8 \mathrm{wpc}$ (Figure 7D), with similar findings at 10 wpc (data not shown). Overnight in vitro culture at 8 wpc demonstrated that these early corticotrophs secreted abundant ACTH, which was suppressible by dexamethasone (Table 1).

Finally, with the onset of major differentiation of the external genitalia at $8 \mathrm{wpc}$ (Figure 8), we determined the capacity of the fetal adrenal gland to secrete androgens. Androstenedione and testosterone were assayed from the media of paired adrenal

\section{Figure 6}

Cortisol content and secretion from the early human adrenal cortex. (A) Cortisol content (mean \pm SEM) per mg adrenal tissue at 8,9 , and 10 wpc, with 8 wpc kidney as control. (B) Cortisol secretion (mean \pm SEM) of paired adrenal glands stimulated by $10 \mu \mathrm{M}$ forskolin at 8,9 , and 10 wpc. (C) Cortisol secretion (mean \pm SEM) of paired adrenal glands at $8 \mathrm{wpc}$ in response to varying doses of ACTH(1-24). Statistical analyses of stimulated secretion compared to basal secretion achieved the same level of significance $\left({ }^{* * *} P<0.005\right)$ for each ACTH dose. ${ }^{*} P<0.05$; ${ }^{* *} P<0.02$. (D) PCR amplification of type 2 melanocortin receptor (MC2R) in the presence $(+)$ and absence $(-)$ of $R T$ in the adrenal gland, testis, and ovary at $8 \mathrm{wpc}$ with GAPDH control.
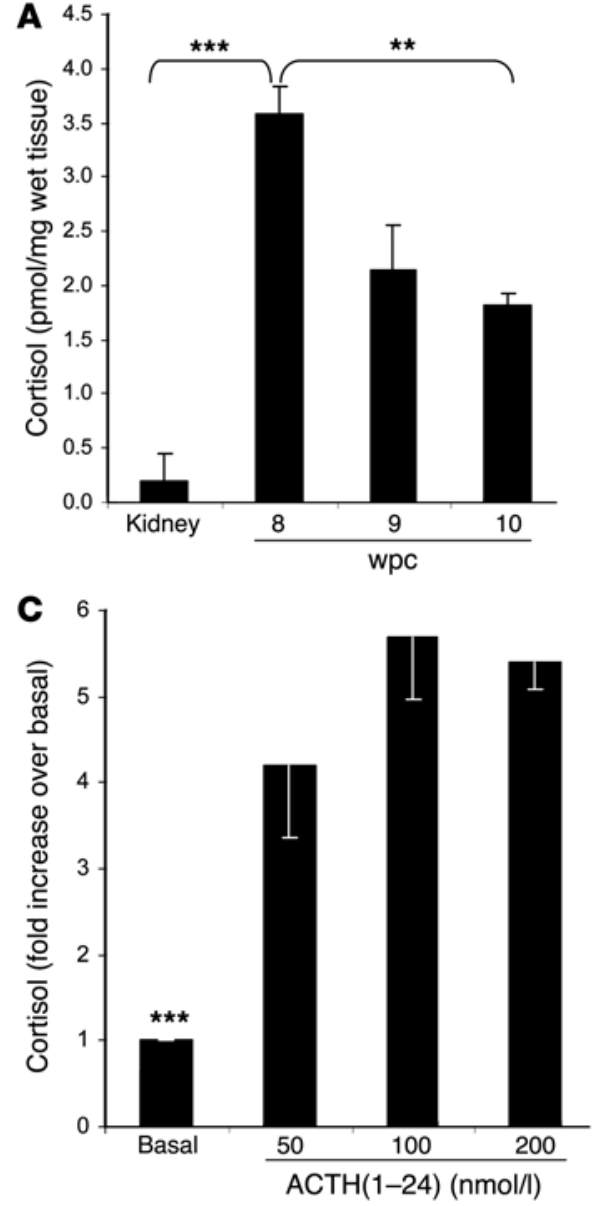

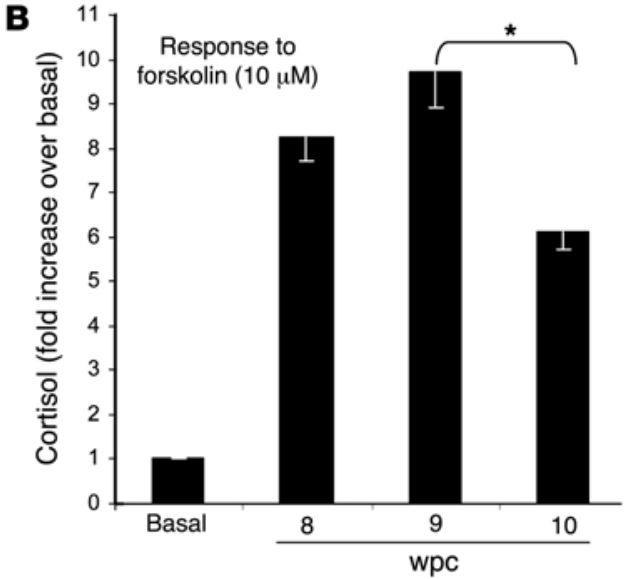

D

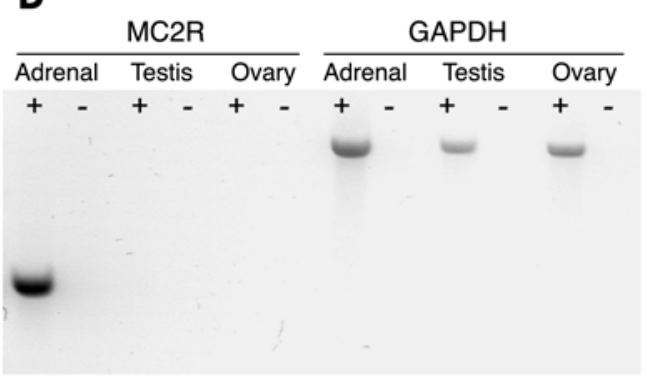




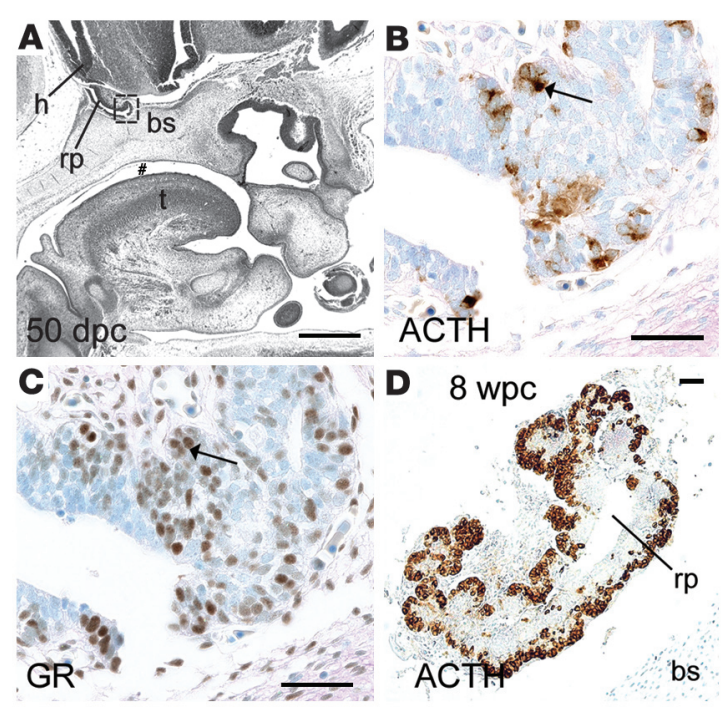

glands cultured overnight in the presence or absence of ACTH, forskolin, or dexamethasone. Dexamethasone failed to alter androstenedione and testosterone secretion significantly, both of which were stimulated approximately 3 -fold in male and female adrenal glands by forskolin and, to a lesser extent, ACTH (Table 2). In contrast, neither ACTH-stimulated coculture of adrenal with fetal testis or ovary nor ACTH stimulation of gonad alone augmented androgen production (data not shown). The conversion of androstenedione to testosterone relied on 17 $\beta$-hydroxysteroid dehydrogenase (HSD17B) activity. By RT-PCR, the type 3 isoform, HSD17B3, responsible for testosterone biosynthesis in the testis, was only very weakly detected in the first-trimester adrenal cortex (Figure 8C). The type 5 enzyme, encoded by the AKR1C3 gene, was more readily detected. The detection of both androstenedione and testosterone was validated by mass spectrometry (Supplemental Figure 1).

\section{Discussion}

Sexual differentiation of the external genitalia is a fundamental aspect of human development, tied by the phenotype of CYP2 1 deficiency to the fetal adrenal cortex. The nature and timing of this event are important. From the second trimester onward, adrenocortical steroids such as dehydroepiandrosterone can act as precursors for androgen biosynthesis $(3,11)$. However, by this stage, the female fetus is relatively well protected, as high placental aromatase activity converts this androgen to estrogen (12). In contrast, sexual dimorphism is established during a critical period in the first trimester, when a relative lack of aromatase permits normal androgen-mediated male sexual differentiation (see Figure 8) but conversely renders the female fetus susceptible to virilization (11). We have investigated this key period of human endocrine development.

Adrenal enzyme expression by 50-52 dpc included those that theoretically might convert placental progesterone to cortisol. This activity has been noted later in human gestation (13); however, in studies of other primates, progesterone accounted for less than $5 \%$ of circulating cortisol $(14,15)$. Similarly, during the first trimester, placental transfer of maternal cortisol must be insufficient to suppress ACTH; otherwise the virilized phenotype

\section{Figure 7}

Human early anterior pituitary development. (A) Sagittal section from head at $50 \mathrm{dpc}$. Pound symbol indicates oral cavity. (B-D) Brightfield IHC with antibodies to ACTH (B and D) and GR (C) counterstained by toluidine blue. $\mathbf{B}$ and $\mathbf{C}$ show higher-magnification views of boxed region in A. Arrows show overlapping expression profiles of cytoplasmic ACTH and nuclear GR in adjacent sections. (D) Anterior pituitary at 8 wpc. bs, basosphenoid bone; h, developing hypothalamus; rp, Rathke's pouch; t, tongue. Scale bars: $500 \mu \mathrm{m}$ (A), $100 \mu \mathrm{m}$ (B and C), $200 \mu \mathrm{m}$ (D).

of CYP21 deficiency would never arise. Adrenal generation of cortisol de novo needs HSD3B2 activity. Previously it has only been detected much later during gestation, particularly near parturition, where glucocorticoid biosynthesis ensures lung maturity (3). In this study, the unexpected transient presence of HSD3B2 correlated to the expression of its regulatory orphan nuclear receptor, NGFI-B (8), and cortisol biosynthesis that peaked at 8-9 weeks of human development.

Adrenal regulation during the first trimester has been hypothetical. ACTH from fetal corticotrophs has obviously been a strong candidate. However, several inconsistencies have clouded its role (3): adrenal development prior to $15 \mathrm{wpc}$ appeared normal in anencephaly, where fetuses lack the anterior pituitary; mid- and late-gestation dehydroepiandrosterone biosynthesis is not correlated to circulating ACTH concentrations; fetal zone involution in the newborn period ensues in the presence of relatively constant $\mathrm{ACTH}$; and the precise onset of anterior pituitary function has never been correlated to that of the adrenal cortex. Our data illustrate that the anterior pituitary-adrenal axis does indeed appear mature during this early developmental window. The development of anterior pituitary corticotrophs and the adrenal gland was contemporaneous with ACTH detected and robustly secreted in the same fetuses where adrenocortical enzyme expression commenced. Active circulation and blood vessels, described earlier than in previous studies (16), could provide a means of delivering ACTH to the adrenal gland, which responded with increased cortisol biosynthesis. Superimposed on these findings, the transient presence of NGFI-B would determine steroidogenic preference by regulating HSD3B2 expression in much the same manner as recently discovered during late gestation (8). However, the question remains of what determines the intriguing expression profile of NGFI-B.

Our experiments also investigated the potential secretion of androgens by the early human adrenal gland. During the first

\section{Table 1}

ACTH secretion in vitro from the anterior pituitary at $8 \mathrm{wpC}$

\section{ACTH content \\ Control \\ Basal \\ $<1.1 \mathrm{pmol} / / / 16 \mathrm{~h}$ \\ Inhibition by dexamethasone \\ $5 \mathrm{nM}$ \\ $1 \mu \mathrm{M}$ \\ $32.34 \% \pm 13.0 \%$ \\ $51.9 \% \pm 2.5 \%$}

Control, media alone; Basal, after overnight culture of anterior pituitary. Percentage decrease in media ACTH upon exposure to dexamethasone was standardized against untreated anterior pituitary explant. Normal upper limit of circulating ACTH in human adults is $\sim 9 \mathrm{pmol} / \mathrm{l}$. Results shown as mean \pm SEM from at least 2 experiments. 


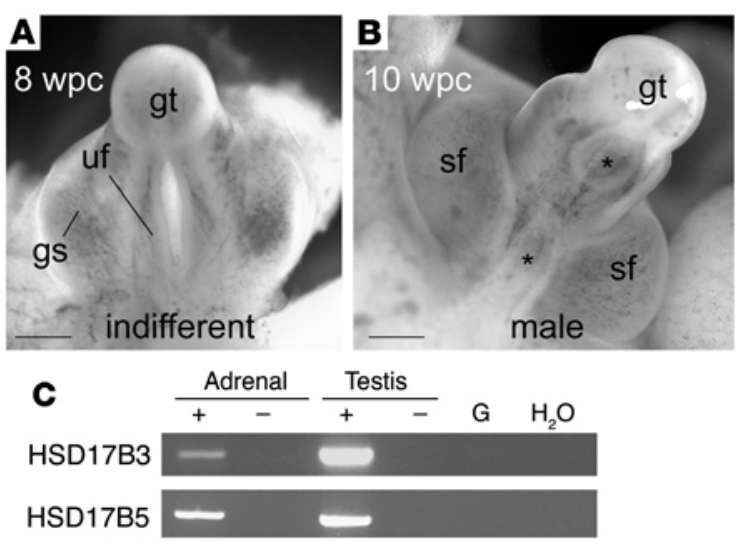

Figure 8

Virilization of the external genitalia and adrenal androgen biosynthesis. (A) Undifferentiated human external genitalia at 8 wpc. (B) Male differentiation of scrotal folds and fusion of the urethral folds (asterisks mark patent regions, either side) at 10 wpc. gs, genital swelling; gt, genital tubercle; sf, scrotal folds; uf, urethral folds. Scale bars: $500 \mu \mathrm{m}$. (C) PCR (35 cycles) in the presence (+) or absence (-) of RT for HSD17B isoforms known to convert androstenedione to testosterone. HSD17B5 is also known as AKR1C3.

trimester, androstenedione and testosterone secretion could be assayed from a single adrenal gland and (more so testosterone) was ACTH responsive. Although high concentrations of ACTH (as occur in untreated CYP21 deficiency) stimulated testicular androgen biosynthesis in the fetal mouse (17), this was not observed from either testis or ovary of human fetuses. Furthermore, expression of the receptor for ACTH was restricted entirely to the developing adrenal cortex. For HSD17B activity to convert androstenedione to testosterone, the type 5 isoform (also named AKR1C3 as part of the aldo-keto-reductase family) was more readily detected than HSD17B3. Both isoforms were less prevalent in the adrenal gland than in the testis. Nevertheless, the adrenal androgen production that we have observed is perfectly timed to affect the external genitalia (Figure 8). Our current interest is in determining the entire extent of androgen biosynthesis, particularly in light of the alternative pathway recently discovered for gonadal androgens in the pouch young of the Tamar wallaby (18-20).

Clinically, to prevent virilization in CYP21 deficiency, dexamethasone needs to be administered very early in gestation (potentially as early as $6 \mathrm{wpc}$; ref. 1). Defining the mechanism behind this early action of dexamethasone is relevant, as its use has attracted controversy over potential long-term postnatal effects $(21,22)$. Lack of clarity over adrenocortical and anterior pituitary corticotroph function has led experts to question how dexamethasone might act at this early developmental age (1). Studying a human adrenocortical cell line did not support an intra-adrenal mechanism (23). In our experiments using the relevant primary tissue, dexamethasone slightly increased cortisol production but did not significantly alter adrenal androgen production. In contrast, the nuclear (and thus presumably ligand-occupied) localization of the anterior pituitary GR, the identically timed ACTH and steroid enzyme expression, and the in vitro suppression of ACTH by dexamethasone at $8 \mathrm{wpc}$ are concordant with dexamethasone acting therapeutically at the fetal anterior pituitary.
Taken together, these data reveal what we believe to be novel functioning of the early adrenal cortex and reinforce distinctive aspects of human development (perhaps including other higher primates). Our observations suggest a delicate balance to early female differentiation that is vulnerable to androgen before the protective appearance of placental aromatase (12). However, the early cortisol biosynthesis, facilitated by transient expression of adrenocortical NGFI-B and HSD3B2, would inhibit ACTH production by the anterior pituitary corticotroph. This negative feedback would minimize ACTH-driven androgen secretion to produce a transient mechanism that safeguards the major period of female sexual development (Figure 9). In support of this model, loss of cortisol feedback by inactivation of the GR has previously been associated with disrupted female sexual differentiation and ambiguous genitalia at birth (although the patient also carried 1 disrupted allele of the CYP21 gene, this would be insufficient by itself to generate an intersex phenotype; ref. 24).

Our future experiments will expand upon this developmental model by seeking to understand the regulation and identify the molecular consequences of this early adrenal steroid biosynthesis.

\section{Methods}

Collection of human embryonic and fetal material. Ethical approval for these studies was granted by the Southampton and South West Hampshire Local Research Ethics Committee. The collection and staging of human embryonic and fetal material was carried out with informed consent as described previously (25-27) using the Carnegie classification and fetal foot length to provide a direct assessment of gestational age as dpc or wpc. Organs from 121 fetuses were analyzed. Tissue preparation for gene expression studies has been described previously (10).

IHC. IHC was performed as described previously using trypsin or sodium citrate antigen exposure techniques (25). Sections were incubated with primary antibody (optimal dilution shown in Table 3 ) overnight at $4{ }^{\circ} \mathrm{C}$ and with biotinylated secondary antibodies for 2 hours at $4^{\circ} \mathrm{C}$ according to the manufacturer's instructions (Vector Laboratories). Following incubation for 1 hour at room temperature with streptavidin-horseradish peroxidase conjugate (1:200; Vector Laboratories), the color reaction was developed with diaminobenzidine containing $0.1 \% \mathrm{H}_{2} \mathrm{O}_{2}$, and slides were counterstained with toluidine blue. For brightfield dual IHC, the Vector Red kit was used according to the manufacturer's instructions (Vector Laboratories). All antibodies to steroidogenic enzymes have been reported previ-

Table 2

Adrenal secretion of androstenedione and testosterone at 8 wpc

$\begin{array}{lccc} & \begin{array}{c}\text { Mean } \pm \text { SEM } \\ \text { Androstenedione secretion }\end{array} & \text { Fold increase } & \boldsymbol{P} \\ \text { Basal } & 29.3 \pm 2.77 \mathrm{nmol} / / & - & - \\ \text { Forskolin } & 117 \pm 13.5 \mathrm{nmol} / \mathrm{l} & 4.00 \pm 0.11 & <0.005 \\ \text { ACTH } & 35.0 \pm 1.73 \mathrm{nmol} / / & 1.20 \pm 0.03 & <0.05 \\ \text { DEX } & 22.9 \pm 3.40 \mathrm{nmol} / \mathrm{l} & 0.78 \pm 0.08 & \mathrm{NS} \\ \text { Testosterone production } & & \\ \text { Basal } & 1.15 \pm 0.10 \mathrm{nmol} / \mathrm{l} & - & - \\ \text { Forskolin } & 3.33 \pm 0.33 \mathrm{nmol} / / & 2.90 \pm 0.29 & <0.002 \\ \text { ACTH } & 2.50 \pm 0.10 \mathrm{nmol} / \mathrm{l} & 2.17 \pm 0.09 & <0.001 \\ \text { DEX } & 1.05 \pm 0.23 \mathrm{nmol} / \mathrm{l} & 0.91 \pm 0.20 & \text { NS }\end{array}$

Androstenedione and testosterone were not detected in control media. The response to $10 \mu \mathrm{M}$ forskolin, $100 \mathrm{nM} \mathrm{ACTH}(1-24)$, and $1 \mu \mathrm{M}$ dexamethasone (DEX) was tested. 


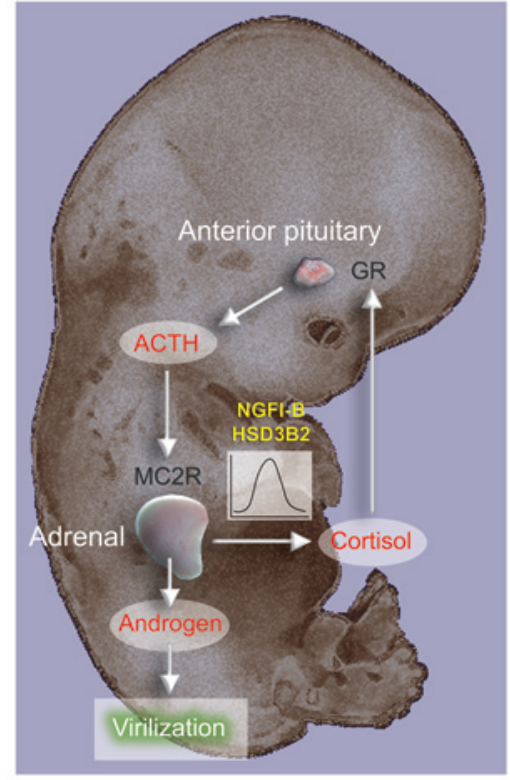

Figure 9

Schematic diagram of human early adrenal function and its implications for androgen-mediated development.

ously with control data $(10,28-36)$. In the current experiments, controls included omission of primary or secondary antibody. The ACTH antibody was raised against amino acids 1-24 of ACTH [ACTH(1-24)]. Potential cross-reactivity was limited to $\alpha-\mathrm{MSH}$.

$R T-P C R$. Total RNA was isolated from tissues using Tri-Reagent (SigmaAldrich), and cDNA was synthesized from $1 \mu \mathrm{g}$ per sample with Superscript III (Invitrogen Corp.). Where possible, intron-spanning primer pairs were designed for the amplification of sequenced products (Supplemental Table 1). For the single-exon $M C 2 R$ gene, the control sample lacked RT.

Tissue culture. Adrenal glands were cultured as organs overnight in DMEM/ F12 (PAA Laboratories Inc.) supplemented with $2 \%$ Ultroser SF (i.e., steroid free; BioSepra) and $1 \%$ ITS $(\mathrm{BD})$ at $37^{\circ} \mathrm{C}$ in humidified $5 \% \mathrm{CO}_{2}$ and $95 \%$ air. This media composition has proved optimal in our experience for the maintenance of phenotype of the human adrenocortical tumor cell line NCI-H295R $(37,38)$. Anterior pituitary was cultured overnight in the same media (basal analysis). For analysis of glucocorticoid feedback, the gland, dissected from the developing sella turcica, was divided in the midline for culture as 2 explants. Following initial overnight culture and media ACTH assay, fresh media was added containing either vehicle or dexamethasone (Sigma-Aldrich) for a second overnight incubation. Media ACTH assay results were expressed as percent inhibition following the addition of dexamethasone standardized against the untreated sample.
Steroid extraction, hormone assay, and verification. For organ steroid analysis, weighed adrenal glands were ground in liquid nitrogen, suspended in phosphate-buffered saline ( $\mathrm{pH} 7.2$ ), and extracted in dichloromethane $(2.0 \mathrm{ml})$. After brief centrifugation, the organic phase was isolated and extracts were dried under air at $25^{\circ} \mathrm{C}$ and resuspended in assay diluent $(250 \mu \mathrm{l})$. Media steroid and ACTH assays were performed directly on $0.5 \mathrm{ml}$ aliquots from culture. Hormones were assayed according to the manufacturer's instructions using Immulite 2000 (ACTH, androstenedione and cortisol; Diagnostic Products Corporation) and ADVIA Centaur (testosterone; Bayer) automated chemiluminescent immunoassays. The ACTH assay targets 2 sites and only detects intact ACTH (amino acids 1-39). Cortisol, testosterone, androstenedione, and ACTH were undetected in control media samples. Assay cross-reactivity was minimal: 5.4\% for testosterone with $5 \alpha$-dihydrotestosterone, $1.49 \%$ for androstenedione with testosterone, and less than $1 \%$ for other known androgenic steroids. Gas chromatography/mass spectrometry was used to verify the steroid immunoassays (Supplemental Figure 1).

Statistics. Steroid assays were analyzed using the 2-tailed Student's $t$ test. Adrenal steroid experiments were conducted at least 3 times at each dose and age on either paired or pooled organs. ACTH was measured on 2 (dexamethasone studies) or 3 occasions (basal studies). Results are presented as mean \pm SEM.

\section{Acknowledgments}

Neil Hanley is a UK Department of Health Clinician Scientist. This work was funded by the University of Southampton. The authors are very grateful to Cedric Shackleton for facilitating the steroid validation experiments and to Anne Chad and colleagues at the Princess Anne Hospital for the collection of human fetal material. We would also like to thank the investigators who gifted antibodies.

Received for publication March 21, 2005, and accepted in revised form January 3, 2006.

Address correspondence to: Neil A. Hanley, Human Genetics Division, Duthie Building, Mailpoint 808, Southampton General Hospital, Tremona Road, Southampton, SO16 6YD, United Kingdom. Phone: 44-23-8079-5040; Fax: 44-23-8079-4264; E-mail: N.A.Hanley@soton.ac.uk.

\section{Table 3}

Primary antibodies

$\begin{array}{lcc}\text { Primary antibody } & \text { Raised in } & \text { Dilution } \\ \text { Polyclonal anti-StAR } & \text { Sheep } & 1: 3,000 \\ \text { Polyclonal anti-CYP11A } & \text { Rabbit } & 1: 1,500 \\ & & \\ \text { Polyclonal anti-CYP17 } & \text { Rabbit } & 1: 1,000 \\ \text { Polyclonal anti-HSD3B } & \text { Rabbit } & 1: 500 \\ \text { Polyclonal anti-CYP21 } & \text { Rabbit } & 1: 7,500 \\ & & \\ \text { Polyclonal anti-CYP11B1/2 } & \text { Rabbit } & 1: 500 \\ & & \\ \text { Monoclonal anti-ACTH } & \text { Mouse } & 1: 1,000 \\ \text { Monoclonal anti-GR } & \text { Mouse } & 1: 20 \\ \text { Monoclonal anti-CD34 } & \text { Mouse } & 1: 50 \\ \text { Monoclonal anti-CK19 } & \text { Mouse } & 1: 100 \\ \text { Monoclonal anti-Ki67 } & \text { Mouse } & 1: 200 \\ \text { Polyclonal anti-NGFI-B } & \text { Rabbit } & 1: 200\end{array}$

\section{Source and/or ref.}

(28)

Gift from A. Payne (Stanford University, Stanford, California, USA; refs. 29, 30)

$$
\begin{aligned}
& (10,31,32) \\
& (33,34,35)
\end{aligned}
$$

Gift from B.-C. Chung (University of Taipei, Taipei, Republic of China; ref. 36)

Gift from H. Takemori and M. Okamoto (Osaka University School of Medicine, Osaka, Japan)

\section{Neomarkers}

Alexis Corp.

Novocastra Ltd.

Novocastra Ltd.

Novocastra Ltd.

Geneka Biotechnology Inc. (8) 
1. White, P.C., and Speiser, P.W. 2000. Congenital adrenal hyperplasia due to 21-hydroxylase deficiency. Endocr. Rev. 21:245-291.

2. David, M., and Forest, M.G. 1984. Prenatal treatment of congenital adrenal hyperplasia resulting from 21-hydroxylase deficiency. J. Pediatr. 105:799-803.

3. Mesiano, S., and Jaffe, R.B. 1997. Developmenta and functional biology of the primate fetal adrenal cortex. Endocr. Rev. 18:378-403.

4. Pepe, G.J., and Albrecht, E.D. 1990. Regulation of the primate fetal adrenal cortex. Endocr. Rev. 11:151-176.

5. Narasaka, T., Suzuki, T., Moriya, T., and Sasano, H. 2001. Temporal and spatial distribution of corticosteroidogenic enzymes immunoreactivity in developing human adrenal. Mol. Cell. Endocrinol. 174:111-120.

6. Baker, B.L., and Jaffe, R.B. 1975. The genesis of cell types in the adenohypophysis of the human fetus as observed with immunocytochemistry. Am.J. Anat. 143:137-161.

7. Gray, E.S., and Abramovich, D.R. 1980. Morphologic features of the anencephalic adrenal gland in early pregnancy. Am. J. Obstet. Gynecol. 137:491-495.

8. Bassett, M.H., et al. 2004. The orphan nuclear receptor NGFIB regulates transcription of 3betahydroxysteroid dehydrogenase. implications for the control of adrenal functional zonation. J. Biol. Chem. 279:37622-37630.

9. Hanley, N.A., et al. 1999. Expression of steroidogenic factor 1 and Wilms' tumour 1 during early human gonadal development and sex determination. Mech. Dev. 87:175-180.

10. Hanley, N.A., Rainey, W.E., Wilson, D.I., Ball, S.G., and Parker, K.L. 2001. Expression profiles of SF-1, DAX1, and CYP17 in the human fetal adrenal gland: potential interactions in gene regulation. Mol. Endocrinol. 15:57-68.

11. Grumbach, M.M., and Auchus, R.J. 1999. Estrogen: consequences and implications of human mutations in synthesis and action. J. Clin. Endocrinol. Metab. 84:4677-4694.

12. Kitawaki, J., et al. 1992. Increasing aromatase cytochrome P-450 level in human placenta during pregnancy: studied by immunohistochemistry and enzyme-linked immunosorbent assay. Endocrinol ogy. 130:2751-2757.

13. Macnaughton, M.C., Taylor, T., McNally, E.M., and Coutts, J.R. 1977. The effect of synthetic ACTH on the metabolism of [4-14C]-progesterone by the previable human fetus. J. Steroid Biochem. 8:499-504.

14. Pepe, G.J., and Albrecht, E.D. 1980. The utilization of placental substrates for cortisol synthesis by the baboon fetus near term. Steroids. 35:591-597.

15. Ducsay, C.A., Stanczyk, F.Z., and Novy, M.J. 1985. Maternal and fetal production rates of progesterone in rhesus macaques: placental transfer and conversion to cortisol. Endocrinology. 117:1253-1258.

16. McClellan, M., and Brenner, R.M. 1981. Development of the fetal adrenals in non-human primates: electron microscopy. In Fetal endocrinology. M.J. Novy and M.J. Resko, editors. Academic Press. New York, New York, USA. 383-403.

17. O'Shaughnessy, P.J., et al. 2003. Adrenocorticotropic hormone directly stimulates testosterone production by the fetal and neonatal mouse testis. Endocrinology. 144:3279-3284.

18. Auchus, R.J. 2004. The backdoor pathway to dihydrotestosterone. Trends Endocrinol. Metab. 15:432-438.

19. Wilson, J.D., Leihy, M.W., Shaw, G., and Renfree, M.B. 2003. Unsolved problems in male physiology: studies in a marsupial. Mol. Cell. Endocrinol. 211:33-36.

20. Wilson, J.D., et al. 2003. 5alpha-androstane3alpha,17beta-diol is formed in tammar wallaby pouch young testes by a pathway involving 5 alphapregnane-3alpha,17alpha-diol-20-one as a key intermediate. Endocrinology. 144:575-580.

21. Miller, W.L. 1999. Dexamethasone treatment of congenital adrenal hyperplasia in utero: an experimental therapy of unproven safety. J. Urol. 162:537-540.

22. Ritzen, E.M. 2001. Prenatal dexamethasone treatment of fetuses at risk for congenital adrenal hyperplasia: benefits and concerns. Semin. Neonatol. 6:357-362.

23. Dardis, A., and Miller, W.L. 2003. Dexamethasone does not exert direct intracellular feedback on steroidogenesis in human adrenal NCI-H295A cells. J. Endocrinol. 179:131-142.

24. Mendonca, B.B., et al. 2002. Female pseudohermaphroditism caused by a novel homozygous missense mutation of the GR gene. J. Clin. Endocrinol. Metab. 87:1805-1809.

25. Piper, K., et al. 2004. Beta cell differentiation during early human pancreas development. J. Endocrinol. 181:11-23.

26. Bullen, P., and Wilson, D.I. 1997. The Carnegie staging of human embryos: a practical guide. In Molecular genetics of early human development. T. Strachan, D.I. Wilson, and S. Lindsay, editors. BIOS Scientific. Herndon, Viriginia, USA. 265 pp.

27. O'Rahilly, R., and Muller, F. 1987. Developmental stages in human embryos. Carnegie Institution of Washington. Washington, DC, USA. 306 pp

28. Ivell, R., et al. 2000. Acute regulation of the bovine gene for the steroidogenic acute regulatory protein in ovarian theca and adrenocortical cells. J. Mol. Endocrinol. 24:109-118.

29. Anakwe, O.O., and Payne, A.H. 1987. Noncoordinate regulation of de novo synthesis of cytochrome P-450 cholesterol side-chain cleavage and cytochrome P-450 17 alpha-hydroxylase/C17-20 lyase in mouse Leydig cell cultures: relation to steroid production. Mol. Endocrinol. 1:595-603.

30. Garmey, J.C., Guthrie, H.D., Garrett, W.M., Stoler, M.H., and Veldhuis, J.D. 2000. Localization and expression of low-density lipoprotein receptor, steroidogenic acute regulatory protein, cytochrome P450 side-chain cleavage and P450 17-alpha-hydroxylase/C17-20 lyase in developing swine follicles: in situ molecular hybridization and immunocytochemical studies. Mol. Cell. Endocrinol. 170:57-65.

31. Sasano, H., Mason, J.I., and Sasano, N. 1989. Immunohistochemical study of cytochrome P-45017 alpha in human adrenocortical disorders. Hum. Pathol. 20:113-117.

32. Sasano, H., Mason, J.I., and Sasano, N. 1989. Immunohistochemical analysis of cytochrome P-450 17 alpha-hydroxylase in pig adrenal cortex, testis and ovary. Mol. Cell. Endocrinol. 62:197-202.

33. Doody, K.M., et al. 1990. 3 beta-hydroxysteroid dehydrogenase/isomerase in the fetal zone and neocortex of the human fetal adrenal gland. Endocrinology. 126:2487-2492.

34. Sasano, H., Mori, T., Sasano, N., Nagura, H., and Mason, J.I. 1990. Immunolocalization of 3 betahydroxysteroid dehydrogenase in human ovary. J. Reprod. Fertil. 89:743-751.

35. Gell, J.S., et al. 1998. Adrenarche results from development of a 3beta-hydroxysteroid dehydrogenasedeficient adrenal reticularis. J. Clin. Endocrinol. Metab. 83:3695-3701.

36. Hu, M.C., and Chung, B.C. 1990. Expression of human 21-hydroxylase (P450c21) in bacterial and mammalian cells: a system to characterize normal and mutant enzymes. Mol. Endocrinol. 4:893-898.

37. Rainey, W.E., et al. 1993. Regulation of human adrenal carcinoma cell (NCI-H295) production of C19 steroids. J. Clin. Endocrinol. Metab. 77:731-737.

38. Bird, I.M., et al. 1993. Human NCI-H295 adrenocortical carcinoma cells: a model for angiotensinII-responsive aldosterone secretion. Endocrinology. 133:1555-1561. 